wir einsetzen, verstärken die Insulinsekretion, sind also auf den Diabetes Typ 2 ausgerichtet. Aber sie setzen nicht an der zugrundeliegenden Ursache an. Deshalb müssen Diabetiker bisher ja so viele verschiedene Medikamente einnehmen, die den Blutdruck senken, die Lipide senken und und und. Die eine goldene Pille dagegen haben wir nicht. Das ist es, was wir wollen.

Es gibt auch eine weitere Hypothese, welcher Mechanismus hinter der Entstehung eines Diabetes Typ 2 steckt; nämlich, dass ein zu hoher Insulinspiegel an sich, und zwar im Nüchternzustand, die zugrundliegende Ursache ist. Also die Hyperinsulinämie und nicht wie in der klassischen Sicht die Betazell-Dysfunktion soll demnach der Krankheitsauslöser sein. Auch Diabetiker im Endstadium haben offenbar hohe Insulinspiegel. Was sagen Sie dazu?

Ulf Smith: Diese Hypothese geistert schon länger herum, sie wurde aber nie genauer erläutert. Dass die Hyperinsulinämie an sich negative Konsequenzen mit sich bringen kann, ist keine neue Idee. Das muss nicht unbedingt etwas mit der Pathogenese des Diabetes zu tun haben. So weiß man schon seit längerem, dass Insulin die sympathischen Nerven angreifen kann; oder dass es die Sekretion gewisser Leberenzyme negativ beeinflusst, wodurch beispielsweise eine Fibrinolyse ausgelöst wird. Insulin kann auch die Natriumsekretion derartig beeinflussen, dass der Blutdruck steigt. Klar, die Hyperinsulinämie an sich kann sich negativ auswirken, aber wie genau, daran wird noch geforscht.
Wenn die Hyperinsulinämie negative Konsequenzen haben kann, sollte man dann nicht die bisherige Diabetestherapie überdenken, die ja u.a. darauf basiert, die Insulinproduktion zu steigern? Ulf Smith: Eine heikle Frage. Die generelle Meinung ist ja bereits, dass es sicher nicht der beste Weg ist, einen Patienten mit Typ2-Diabetes zu behandeln, indem man immer und immer wieder die Betazellen stimuliert, auch aus anderen Gründen nicht. Deshalb geht der Einsatz der Sulfonylharnstoffe zurück. Aber wahrscheinlich nicht, weil die „klinische Welt“ hier an die Konsequenzen der Hyperinsulinämie denkt, sondern daran, dass Sulfonylharnstoffe Hypoglykämien auslösen können. Und weil ihre Wirkung im Gegensatz zu anderen Substanzen nur kurz anhält und dann nach sechs bis zehn Monaten nachlässt.

Und sicherlich sollten wir uns nicht nur auf die Glukose fokussieren. Der Zucker ist nur die Spitze des Eisberges. Viele andere Probleme, die mit einem Typ-2-Diabetes assoziiert sind wie das Krebsrisiko, sind keine Auswirkungen des Zuckers. Hier spielt die Insulinresistenz die Schlüsselrolle. Wir wissen nur nicht genügend darüber. Deshalb kommen wir ja auf Symposien wie dem hier in Bad Homburg zusammen. Wir müssen mehr forschen! Denn Diabetes und Übergewicht nehmen zu. Interview: Veronika Schlimpert

Quelle: Interview im Rahmen des EFSD/Lilly-Symposiums am 2. und 3. Mai 2013 in Bad Homburg

\title{
Lipidtherapie: Muss HDL-Cholesterin jetzt neu bewertet werden?
}

\author{
Lange Zeit war die Welt des Cholesterins sauber in zwei Lager geteilt: das böse LDL- und das gute \\ HDL-Cholesterin. In Bezug auf das HDL stellt sich die Situation inzwischen komplexer dar.
}

\begin{abstract}
Personen mit niedrigem HDL-Spiegel haben ein hohes kardiovaskuläres Risiko - und umgekehrt. Das ist in epidemiologischen Studien eindeutig gezeigt worden. Versuche, durch eine medikamentöse Erhöhung niedriger HDL-Werte das Risiko für Herzinfarkt und Schlaganfall zu senken, sind bislang jedoch gescheitert. In Studien brachte weder Nikotinsäure noch der CETP-Hemmer Dalcetrapib den gewünschten Erfolg. Warum das?

Laut Prof. Klaus Parhofer, Lipidexperte der Ludwig-Maximilians-Universität München, steht es „außer Zweifel“, dass gewisse HDL-Subfraktionen antiatherosklerotisch wirken. Der beobachtete epidemiologische Zusammenhang lasse sich so aber nicht erklären, sondern beruhe eher darauf, dass bei niedrigem HDL-Cholesterin besonders viele atherosklerosefördernde ApoB-haltige Lipoproteine vorhanden seien. „Ein niedriges HDL-Cholesterin ist ein Risikomarker, aber es ist offenbar nicht als Therapie-Target geeignet", sagte Parhofer. Wenn bei einem Patienten ein niedriger HDL-Spiegel $(<40 \mathrm{mg} / \mathrm{dl})$ gemessen wird, muss man trotzdem aktiv werden. „Diese Patienten haben ja ein deutlich erhöhtes kardiovaskuläres Risiko. Das muss man reduzieren, indem man andere Risikofaktoren wie LDL, Blutdruck und Blutzucker optimiert", betonte Parhofer.

Änderungen gibt es auch in der Therapie von Patienten mit erhöhtem Lipoprotein(a). Bei diesen Patienten ist die Behandlung mit Nikotinsäure kausal wirksam. Der Wirkstoff wurde jedoch wegen des Misserfolgs in der HDL-Therapie im Januar vom Markt genommen. Das Vorgehen bei hohem Lp(a) muss sich daher jetzt darauf beschränken, begleitend auftretende Risikofaktoren möglichst gut zu kontrollieren, so Parhofer.
\end{abstract}

Über- und Untertherapie mit Statinen

Die LDL-Cholesterin-Senkung zur Vermeidung kardiovaskulärer Ereignisse gehört zu den am besten belegten Therapien in der Inneren Medizin.
Trotz der hohen Effektivität der Statine ist das Therapieprinzip aber noch nicht ausgeschöpft, so die Einschätzung Parhofers. Es gebe eine „Fehlversorgung" in beide Richtungen: Zum einen werden Patienten mit Statinen behandelt, die zwar einen erhöhten LDL-Spiegel, aber ein niedriges Absolutrisiko aufweisen. Andererseits gibt es Hochrisikopatienten, bei denen eine Fire-and-Forget-Strategie angewendet wird - anstelle einer zielwertorientierten Therapie - und die damit nach derzeitigem Kenntnisstand unzureichend behandelt sind. Die LDL-senkende Therapie wird zudem durch das Auftreten einer Statinintoleranz limitiert. Laut Parhofer leiden etwa 10\% der Statinpatienten an Nebenwirkungen. „In den meisten Fällen sind das muskuläre Beschwerden ohne CK-Erhöhung." Junge, sportliche Patienten mit familiärer Hypercholesterinämie sind davon besonders häufig betroffen. Die Nebenwirkungen würden meistens dazu führen, dass die Therapie nicht in der angestrebten Dosierung fortgesetzt werden könne.

\section{Neue Therapieansätze}

Einige Lücken in der Lipidtherapie hofft man durch neuartige Therapieansätze schließen zu können. Derzeit werden zwei CETP-Hemmer, Anacetrapib und Evacetrapib, in Endpunktstudien untersucht. Im Unterschied zu Dalcetrapib erhöhen sie nicht nur das HDL, sondern senken auch das LDL. Speziell zur Behandlung der homozygoten familiären Hyperlipidämie haben sich Mipomersen und Lomitapid als vielversprechend erwiesen. Den nach Parhofers Einschätzung "global wahrscheinlich interessantesten" Ansatz stellen Antikörper gegen PCSK-9 dar. Sie bewirken, dass der LDL-Rezeptor schneller wieder aktiv und somit LDL beschleunigt abgebaut wird. Derzeit haben mindestens neun Firmen PCSK-9-Antikörper in Entwicklung. Beate Schumacher 